\title{
Case Report: Induced Lactation in a Transgender Woman
}

\author{
Tamar Reisman ${ }^{1,2, *}$ and Zil Goldstein ${ }^{1}$
}

\begin{abstract}
Objective: Our report describes a case of nonpuerperal induced lactation in a transgender woman. Methods: We present the relevant clinical and laboratory findings, along with a review of the relevant literature. Results: A 30-year-old transgender woman who had been receiving feminizing hormone therapy for the past 6 years presented to our clinic with the goal of being able to breastfeed her adopted infant. After implementing a regimen of domperidone, estradiol, progesterone, and breast pumping, she was able to achieve sufficient breast milk volume to be the sole source of nourishment for her child for 6 weeks. This case illustrates that, in some circumstances, modest but functional lactation can be induced in transgender women.
\end{abstract}

Keywords: breastfeeding; gender dysphoria; induced lactation; transgender

\section{History of Present Illness}

A 30-year-old transgender woman presented to clinic seeking help to achieve her goal of breastfeeding. She explained that her partner was pregnant but not interested in breastfeeding, and that she hoped to take on the role of being the primary food source for her infant.

The patient's medical history was significant for gender incongruence for which she initiated a feminizing hormone regimen in 2011. At the time of our first visit, she was taking spironolactone $50 \mathrm{mg}$ po bid, estradiol $2 \mathrm{mg}$ po bid, and micronized progesterone $100 \mathrm{mg}$ po bid. Her medical history was also significant for panic disorder, for which she was taking occasional clonazepam, and insomnia, for which she was taking occasional zolpidem. She was otherwise known to be in good general health and reported no complaints. Apart from her use of oral estradiol, she had no known risk factors for thromboembolism. She did not have a personal or familial history of thromboembolism and was a nonsmoker.

Her surgical history was notable only for laparoscopic cholecystectomy. She had not had any genderaffirming surgeries such as breast augmentation, orchiectomy, or vaginoplasty.
On initial examination the patient was a pleasant, well nourished, well developed woman who appeared her stated age. Her breasts were noted to be Tanner stage V.

Her laboratory results from her initial evaluation included estradiol $119 \mathrm{pg} / \mathrm{mL}$, progesterone $8.70 \mathrm{ng} /$ $\mathrm{mL}$, prolactin $9.5 \mathrm{ng} / \mathrm{nL}$, sex hormone binding globulin $48 \mathrm{nmol} / \mathrm{L}$, and total testosterone $256 \mathrm{ng} / \mathrm{dL}$.

\section{Course}

Previous investigators have reported the following basic framework for nonpuerperal induced lactation: (1) increased estradiol and progesterone dosing to mimic high levels seen during pregnancy, (2) use of a galactogogue to increase prolactin levels, (3) use of a breast pump with the speculation that it would increase prolactin and oxytocin levels, and (4) subsequent reduction in estradiol and progesterone levels, with the intention of mimicking delivery. ${ }^{1-6}$

The patient obtained domperidone from Canada, an antiemetic used off-label as a galactogogue internationally. $^{7}$ The patient was started on domperidone $10 \mathrm{mg}$ po tid. The patient also was given instructions to use her breast pump for $5 \mathrm{~min}$ per breast TID.

${ }^{1}$ Center for Transgender Medicine and Surgery, New York, New York.

${ }^{2}$ Department of Endocrinology, Icahn School of Medicine at Mount Sinai, New York, New York. 
The patient's first follow-up visit occurred at 1 month. On physical examination, she was able to express droplets of milk. The domperidone dose was increased to $20 \mathrm{mg}$ po qid, her micronized progesterone to $200 \mathrm{mg}$ po daily, her estradiol to $8 \mathrm{mg}$ po daily, and her breast pump use to six times daily.

At the 2nd month visit, her progesterone was increased to $400 \mathrm{mg}$ po daily and her estradiol had increased to $12 \mathrm{mg}$ daily.

Three months after starting treatment, 2 weeks before the baby's due date, the patient was making $8 \mathrm{oz}$ of breast milk per day. Her estradiol regimen was shifted to a low dose patch ( $0.025 \mathrm{mg}$ daily) and her progesterone dose was lowered to $100 \mathrm{mg}$ daily.

Three and a half months after she had started the mentioned regimen, the baby was born weighing $6 \mathrm{lbs} 13 \mathrm{oz}$. The patient breastfed exclusively for 6 weeks. During that time the child's pediatrician reported that the child's growth, feeding, and bowel habits were developmentally appropriate. At 6 weeks, the patient began supplementing breastfeedings with $4-8 \mathrm{oz}$ of Similac brand formula daily due to concerns about insufficient milk volume. At the time of this article submission, the baby is approaching 6 months old. The patient continues to breastfeed as a supplement to formula feeding, and she continues to adhere to the medication regimen described earlier.

\section{Discussion}

We believe that this is the first formal report in the medical literature of induced lactation in a transgender woman.

Breastfeeding offers immunological, metabolic, and psychosocial benefits for both mother and infant. Breast milk contains secretory IgA, anti-inflammatory agents, and other immunomodulators that give breastfed infants immunological advantages relative to formula-fed infants. ${ }^{8,9}$

Formula-fed infants are noted to have higher risk of rotavirus infection, otitis media, hospitalization for lower respiratory tract infections, sudden infant death syndrome, necrotizing enterocolitis, asthma, and childhood obesity relative to their breastfed counterparts. ${ }^{8-13}$

In addition, breastfeeding offers economic advantages by allowing families to save resources that might be devoted to formula and infant healthcare. ${ }^{14}$ Women who breastfeed are noted to have lower rates of breast cancer and ovarian cancer than women who have never breastfed. ${ }^{9}$

Breastfeeding has been noted to facilitate motherchild bonding. ${ }^{15}$ For this reason, there has been interest among adoptive parents in inducing lactation, and there have been a number of published articles outlining protocols to that effect. ${ }^{1-6}$

One major difference between the induction of lactation in cis and transgender women is the need for androgen blockade in the latter group. Our patient continued to take spironolactone while breastfeeding for androgen blockade. A known metabolite of spironolactone, cancrenone is excreted in human milk. This has been shown to be $0.2 \%$ of the maternal daily dose, which is thought to be clinically insignificant. ${ }^{16}$ Spironolactone has been reported to have tumorigenic potential in rats, but according to the American Academy of Pediatrics, it is thought to be compatible with breastfeeding. ${ }^{17}$

Throughout this process, the patient used domperidone that was obtained from Canada, where it is commonly used off-label as a galactogogue. Domperidone is not currently available in the United States for gastrointestinal use due to the FDA's concern about its association with cardiac arrhythmias, cardiac arrest, and sudden death when used intravenously. The FDA has warned against domperidone's off-label use as a galactogogue due to its unknown risks on breastfeeding infants. ${ }^{7}$ Although there is significant literature indicating that domperidone increases prolactin levels and milk volume, ${ }^{18-20}$ it is uncertain whether this intervention was necessary to induce lactation. The patient used a breast pump, which likely increased her prolactin levels independently of domperidone. ${ }^{21}$ It is not clear at

Table 1. Patient's Estradiol, Prolactin, and Progesterone Levels at Each Clinic Visit

\begin{tabular}{lcccc}
\hline & $\begin{array}{c}\text { Estradiol } \\
(\mathbf{p g} / \mathrm{mL})\end{array}$ & $\begin{array}{c}\text { Prolactin } \\
(\mathbf{n g} / \mathbf{n L})\end{array}$ & $\begin{array}{c}\text { Progesterone } \\
(\mathbf{n g} / \mathbf{m L})\end{array}$ & $\begin{array}{c}\text { Total } \\
\text { testosterone } \\
(\mathbf{n g} / \mathbf{d L})\end{array}$ \\
\hline Day 0 (first visit) & 63 & 10 & 8 & 20.52 \\
Day 17 & No data & 119 & 13 & No data \\
Day 28 & 129 & 148 & 6 & No data \\
Day 56 & 34 & 143 & 4 & No data \\
Day 70 & 33 & 115 & 5 & No data
\end{tabular}

Female reference ranges: prolactin (nongravid): $1.40-24.00 \mathrm{ng} / \mathrm{nL}$; prolactin (gravid, lactating): no reference range available.

Estradiol (nongravid): follicular phase: $27-122 \mathrm{pg} / \mathrm{mL}$; midcycle phase: 95-433 pg/mL; luteal phase: $49-291 \mathrm{pg} / \mathrm{mL}$; postmenopausal female: $<41 \mathrm{pg} / \mathrm{mL}$.

Estradiol (gravid): first trimester: $188-2497 \mathrm{pg} / \mathrm{mL}$; second trimester: $1278-7192 \mathrm{pg} / \mathrm{mL}$; third trimester: $6137-3460 \mathrm{pg} / \mathrm{mL}$.

Estradiol (lactating): no reference range available.

Progesterone (nongravid): midfollicular phase: $0.31-1.52 \mathrm{ng} / \mathrm{mL}$; midluteal phase: $5.16-18.56 \mathrm{ng} / \mathrm{mL}$; postmenopausal $<0.08-0.78 \mathrm{ng} / \mathrm{mL}$.

Progesterone (gravid): first trimester: $4.73-50.74 \mathrm{ng} / \mathrm{mL}$; second trimester: $19.51-45.30 \mathrm{ng} / \mathrm{mL}$; third trimester: $58.7-214.0 \mathrm{ng} / \mathrm{mL}$.

Progesterone (lactating): no reference range available.

Total testosterone: female: $9-55 \mathrm{ng} / \mathrm{dL}$; female (gravid, lactating): no reference range available. 
this time whether all of the aforementioned components of the patient's medication regimen were necessary to achieve lactation, or whether the patient's hormone levels (as listed in Table 1) were optimized to achieve adequate breast milk volume. Areas of future interest include the optimal dosing of estradiol, progesterone, and galactogogues in inducing lactation, as well as the optimal frequency and duration of pump use. Future investigation will be required to determine the optimal treatment regimen for induced lactation in transgender women.

\section{Acknowledgments}

The authors would like to thank the aforementioned patient for her willingness to share her experience. The authors also thank Mount Sinai Health Systems for their ongoing dedication to meeting the healthcare needs of the LGBTQ community.

\section{Author Disclosure Statement}

No competing financial interests exist.

\section{References}

1. Farhadi R, Roy KP. Induction of lactation in the biological mother after gestational surrogacy of twins: a novel approach and review of literature. Breastfeed Med. 2017;12:373-376.

2. Biervliet FP, Maguiness SD, Hay DM, et al. Induction of lactation in the intended mother of a surrogate pregnancy: case report. Hum Reprod. 2001;16:581-583.

3. Bryant CA. Nursing the adopted infant. Am Board Fam Med. 2006;19:374-379.

4. Anton BF, Garcia-Lara NR, Pallas-Alonson CR. An adoptive mother who became a human milk donor. J Hum Lact. 2017;33:419-421.

5. Szucs KA, Axline SE, Rosenman MB. Induced lactation and exclusive breast milk feeding of adopted premature Twins. J Hum Lact. 2010;26:309-313.

6. Wilson E, Perrin MT, Fogleman A, Chetwynd E. The intricacies of induced lactation for same-sex mothers of an adopted child. J Hum Lact. 2014; 31:64-67.

7. Center for Drug Evaluation and Research. Information by drug class-FDA talk paper: FDA warns against women using unapproved drug, domperidone, to increase milk production. 2016. Available at: www.fda.gov/ Drugs/DrugSafety/InformationbyDrugClass/ucm173886.htm (accessed August 18, 2017).
8. Goldman AS. The immune system in human milk and the developing infant. Breastfeed Med. 2007;2:195-204.

9. Hanson LA, Ahlstedt $S$, Andersson B, et al. Protective factors in milk and the development of the immune system. Pediatrics. 1985;75(1 Pt 2):172-176.

10. Yolken RH, Peterson JA, Vonderfecht SL, et al. Human milk mucin inhibits rotavirus replication and prevents experimental gastroenteritis. J Clin Invest. 1992;90:1984-1991.

11. Ip S, Chung M, Raman G, et al. Breastfeeding and Maternal and Infant Health Outcomes in Developed Countries. Rockville (MD). Agency for Healthcare Research and Quality (US), 2007. (evidence report/technology assessment, No. 153.) Available at: www.ncbi.nlm.nih.gov/books/ NBK38337 (accessed December 2, 2017).

12. Bachrach VR, Schwarz E, Bachrach LR. Breastfeeding and the risk of hospitalization for respiratory disease in infancy: a meta-analysis. Arch Pediatr Adolesc Med. 2003;157:237-243.

13. Arenz S. Breast-feeding and childhood obesity-a systematic review. Int J Obes Relat Metab Disord. 2004;28:1247-1256.

14. United States Breastfeeding Committee. Economic Benefits of Breastfeeding [issue paper]. Raleigh, NC: United States Breastfeeding Committee, 2002.

15. Gribble KD. Mental health, attachment and breastfeeding: implications for adopted children and their mothers. Int Breastfeed J. 2006;1:5.

16. Phelps DL, Karim A. Spironolactone: relationship between concentrations of dethioacetylated metabolite in human serum and milk. J Pharm Sci. 1977;66:1203.

17. Committee on Drugs. American Academy of Pediatrics. The transfer of drugs and other chemicals in human breast milk. Pediatrics. 2001;108: 776-789.

18. Hofmeyr GJ, van Iddekinge B. Domperidone and lactation. Lancet. 1983:647.

19. Brouwers JR, Assies J, Wiersinga WM, et al. Plasma prolactin levels after acute and subchronic oral administration of domperidone and of metoclopramide: a cross-over study in healthy volunteers. Clin Endocrinol (Oxf). 1980;12:435-440.

20. Hofmeyr GJ, Van Iddekinge B, Blott JA. Domperidone: secretion in breast milk and effect on perpheral prolactin levels. Brit J Obs and Gyn. 1985; 92:141-144.

21. Melmed S, Casanueva FF, Hoffman AR, et al. Diagnosis and treatment of hyperprolactinemia: an Endocrine Society clinical practice guideline. JCEM. 2011;96:273-288.

Cite this article as: Reisman T, Goldstein Z (2018) Case report: induced lactation in a transgender woman, Transgender Health 3:1, 24-26, DOI: 10.1089/trgh.2017.0044.

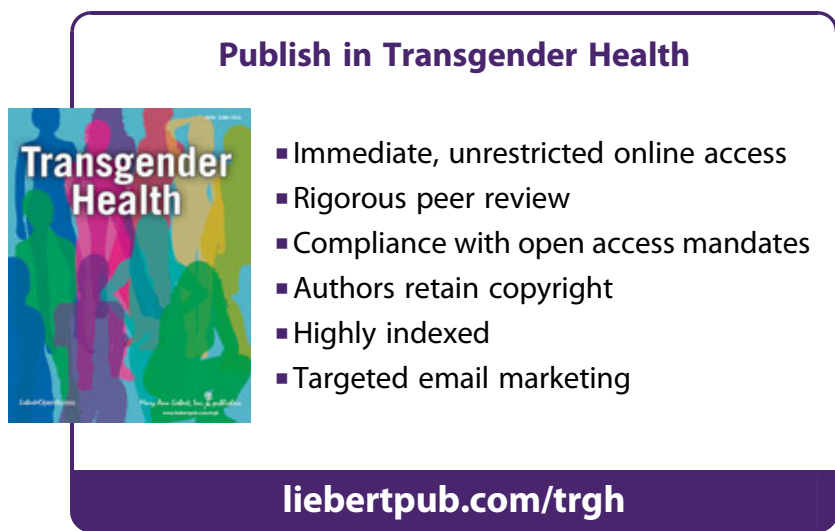

